\title{
Presumed pancreatic cyst proven to be venous malformation due to intestinal malrotation
}
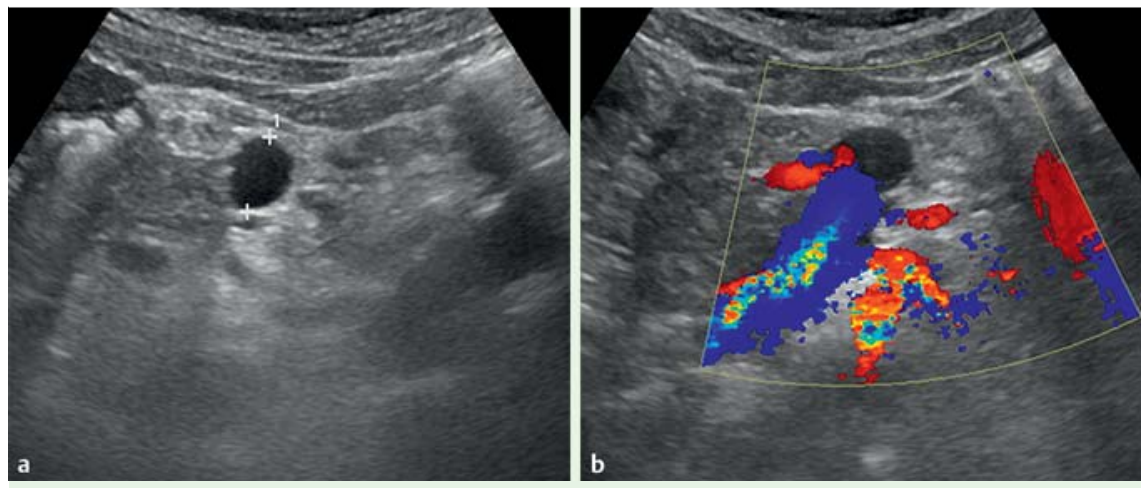

Fig. 1 Abdominal ultrasonography findings. a Benign looking 2-cm sized cystic lesion is seen on pancreas body. $\mathbf{b}$ The cystic lesion is devoid of intraluminal Doppler signal.
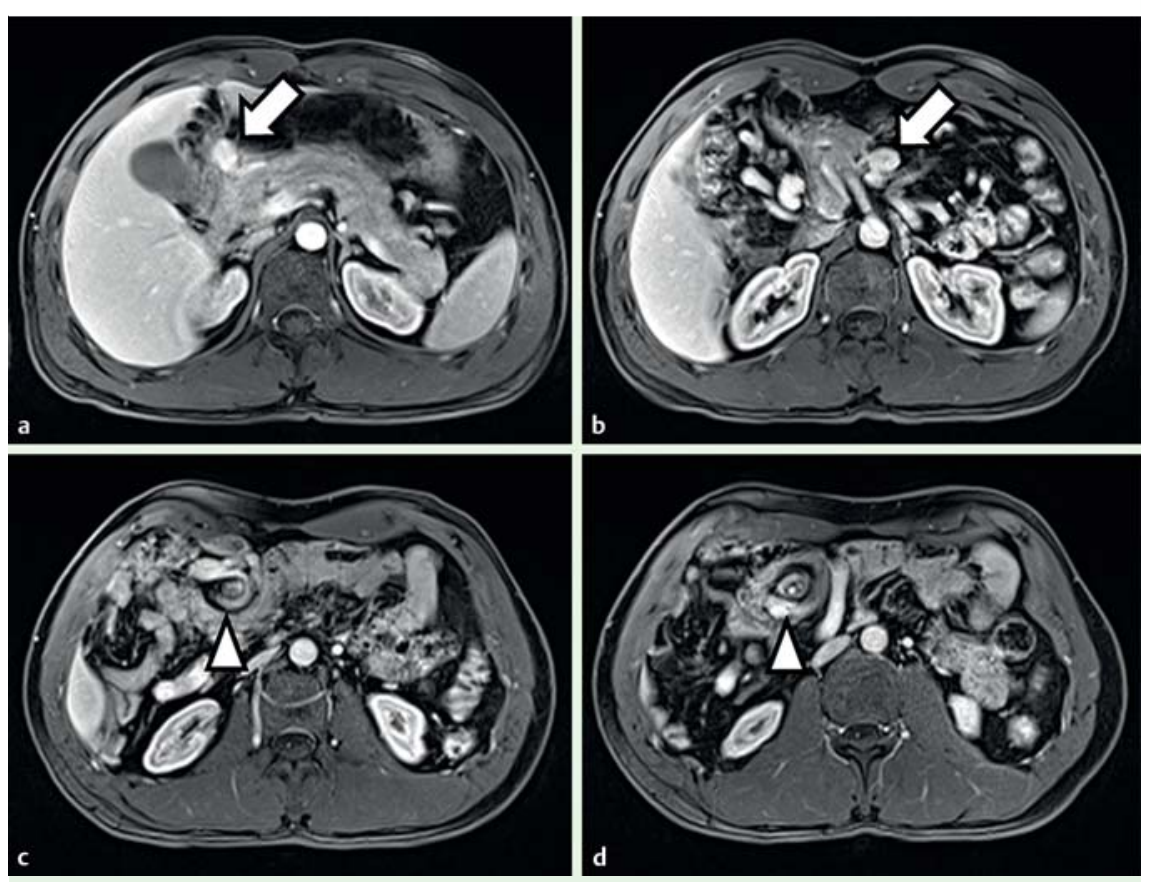

Fig.2 Abdominal magnetic resonance imaging study. a, b Focal venous aneurysms (arrows) are seen at pancreas head (a) and body (b). c, d The whirlpool sign (arrowheads), one of the pathognomonic findings of intestinal malrotation, is clearly demonstrated.

A 27-year-old man was admitted for evaluation of a 20 -year history of chronic epigastric pain and weight loss of $3 \mathrm{~kg}$ over the preceding month. Despite several upper endoscopy examinations, no specific cause could be found. Physical examination showed mild epigastric tenderness. Laboratory findings were unremarkable apart from elevated creatinine level $(3.17 \mathrm{mg} / \mathrm{dL})$ due to focal segmental glomerulosclerosis. To investigate the cause of the abdominal pain, which became aggravated after a meal and improved with fasting, abdominal sonography was performed and showed a benign-looking 2-cm cystic lesion on the body of the pancreas ( $\bullet$ Fig. 1 a). Although the cyst was devoid of intraluminal Doppler signal ( $\bullet$ Fig. $\mathbf{1 b}$ ), it proved on abdominal magnetic resonance imaging (MRI) to be one of two focal venous aneurysms ( $\bullet$ Fig. 2a, b). Twisting of mesenteric vessels along the mesentery was also visualized on abdominal MRI ( $\bullet$ Fig. $\mathbf{2 c}$, d).
Thus, both the focal venous aneurysms and the persistent abdominal pain could be attributable to intestinal malrotation. The findings of a small-bowel series were also compatible with intestinal malrotation: the distal duodenum and jejunum were arranged in a corkscrew appearance on the right side of the ligament of Treitz ( $\bullet$ Fig. 3 a), and the cecum was also located in the right upper quadrant ( $\boldsymbol{Q}$ Fig. $\mathbf{3 b}$ ) [1]. Surgical management was planned, and on laparotomy the small intestine was found to be arranged in a coiled fashion ( $\bullet$ Fig.4a). Ladd's band could clearly be demonstrated and was excised to resolve the intestinal malrotation $(\bullet$ Fig. 4b) [2].

Intestinal malrotation is a congenital anomaly of rotation of the intestine during embryonic development and occurs when the intestine does not make as many turns as it should [3]. It is most often recognized in the neonatal period and is rarely seen in adults. Since venous malformations due to intestinal malrotation can falsely manifest as pancreatic cysts, incidentally found pancreatic cysts in patients with abdominal pain should not be overlooked and additional imaging studies are necessary for further investigation.

Endoscopy_UCTN_Code_CCL_1AC_2AH

Competing interests: None

\section{Young Ju Na, Jong Jin Hyun, Seung Young Kim, Sung Woo Jung, Ja Seol Koo, Hyung Joon Yim, Sang Woo Lee}

Division of Gastroenterology and Hepatology, Department of Internal Medicine, Korea University College of Medicine, Seoul, Korea

\section{References}

1 Long FR, Kramer SS, Markowitz RI et al. Radiographic patterns of intestinal malrotation in children. Radiographics 1996; 16: 547-556; discussion 556-560

2 Prasil P, Flageole H, Shaw KS et al. Should malrotation in children be treated differently according to age? J Pediatr Surg 2000; 35 : $756-758$

3 Torres AM, Ziegler MM. Malrotation of the intestine. World J Surg 1993; 17: 326-331

Bibliography

Dol http://dx.doi.org/

10.1055/s-0034-1377399

Endoscopy 2014; 46: E399-E400

(c) Georg Thieme Verlag KG

Stuttgart · New York

ISSN 0013-726X 


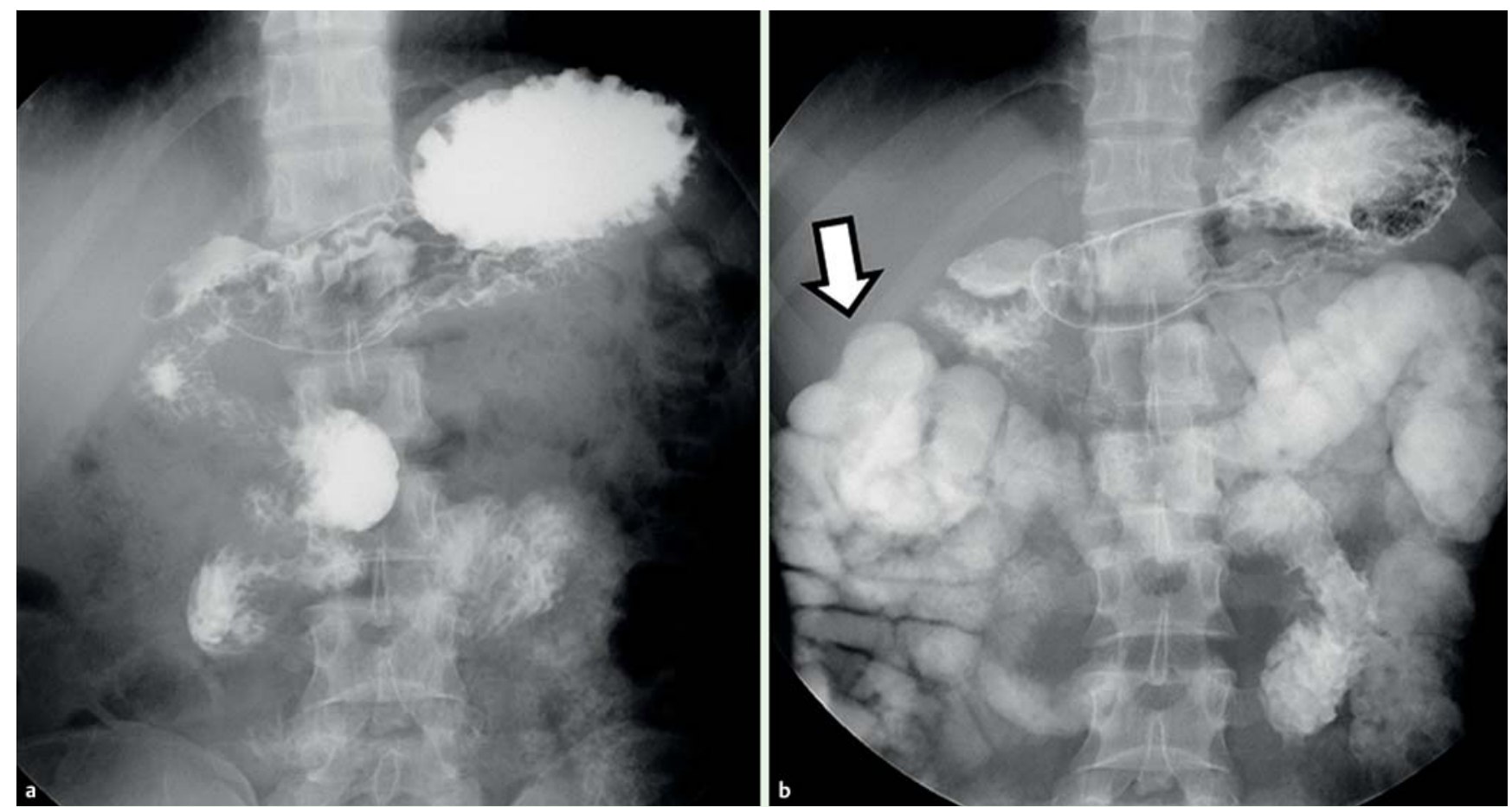

Fig. 3 Upper gastrointestinal series. a The distal duodenum and jejunum are arranged in a corkscrew appearance on the right side of the ligament of Treitz. b The cecum (arrow) is also located in the right upper quadrant.

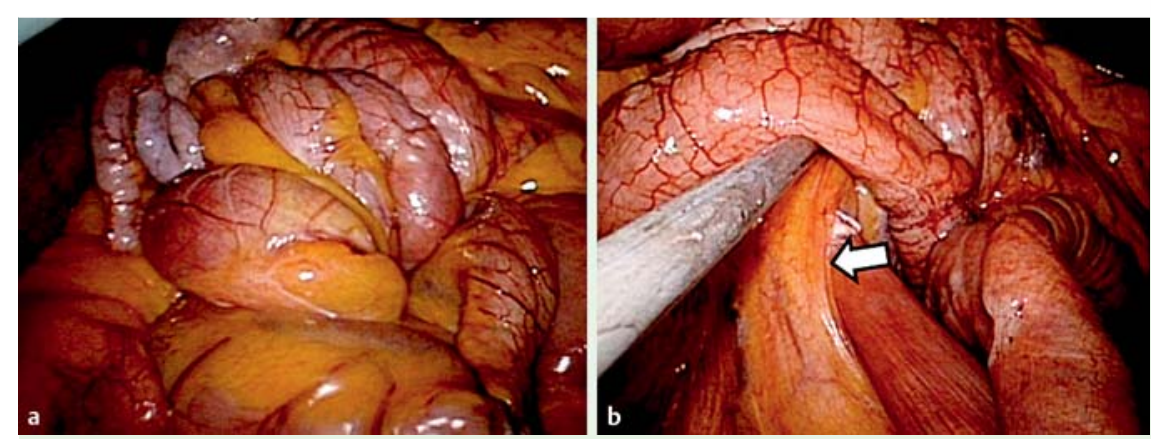

Corresponding author

\section{Jong Jin Hyun, MD, PhD}

Division of Gastroenterology and Hepatology

Department of Internal Medicine

Korea University Ansan Hospital

123, Jeokgeum-ro, Danwon-gu

Ansan-si, Gyeonggi-do

425-707 Korea

Fax: +82-31-4125582

sean4h@korea.ac.kr

Fig. 4 Ladd procedure. a The small intestine is arranged in a coiled fashion. $\mathbf{b}$ Ladd's band (arrow). Excision of this band is the treatment for intestinal malrotation. 